\title{
Novel Treatments for Thymoma and Thymic Carcinoma
}

\author{
Arun Rajan $^{1 *}$, Heather Wakelee ${ }^{2}$ and Giuseppe Giaccone ${ }^{3}$ \\ ${ }^{1}$ Thoracic and Gastrointestinal Oncology Branch, National Cancer Institute, National Institutes of Health, Bethesda, MD, USA, \\ ${ }^{2}$ Department of Medicine, Division of Medical Oncology, Stanford University, Stanford, CA, USA, ${ }^{3}$ Lombardi Comprehensive \\ Cancer Center, Georgetown University, Washington, DC, USA
}

Keywords: thymic epithelial tumors, autoimmune paraneoplastic disorders, genomic changes, anti-cytokine antibodies, biological therapies

The rarity of thymic epithelial tumors (TETs) creates unique challenges in conducting translational research and developing newer paradigms of treatment (1). Nevertheless, steady progress in genome research, the development of thymic cell lines, and an increase in collaborative efforts between various institutions have helped in fostering an increase in the understanding of the biology of TETs and associated paraneoplastic syndromes and given an impetus to the development of newer treatment options for patients with these rare tumors.

Within the last few years, a number of unique genomic changes have been described in TETs (2). Thymic carcinomas have been found to frequently harbor mutations in epigenetic regulatory genes (3). A specific mutation in GTF2I on chromosome 7 is present at a high frequency in World Health Organization (WHO) subtype $\mathrm{A}$ and $\mathrm{AB}$ thymomas (4). A nine-gene signature has been developed to predict the metastatic behavior of thymomas (5). The ongoing study of thymoma as part of The Cancer Genome Atlas (TCGA) project promises to add significantly to the existing body of knowledge about genomic alterations in TETs.

The association between thymomas and autoimmune paraneoplastic disorders is well recognized (6). Some patients with thymoma are also more susceptible to infectious complications due to underlying immune dysregulation (7). Various pathogenic mechanisms implicated in the development of immune dysfunction include decreased expression of the autoimmune regulator (AIRE) gene and the presence of anti-cytokine antibodies (8-13). These discoveries have influenced the screening and management paradigms for patients with thymoma.

Treatment strategies for newly diagnosed and recurrent TETs have also evolved over time. Surgery is considered the cornerstone of management of early stage TETs and complete resection of the tumor has a major impact on prognosis (14). For locally advanced disease, thymectomy with en bloc removal of all involved structures is indicated (15). Active areas of investigation focusing on the surgical management of TETs include an evaluation of minimally invasive surgery and an assessment of the role of surgery for recurrent TETs (16-19). Though of unproven benefit and with some controversy, post-operative radiotherapy is recommended after resection of stage III and IVA TETs, and can be considered for stage II thymic carcinoma and cases of stage II thymoma at high risk for recurrence (WHO B3 histology; extensive transcapsular invasion) (15, 20, 21). Chemotherapy is used for induction therapy in cases of stage III/IVA TETs and for treatment of unresectable and recurrent disease (15). There is scant evidence to support the use of chemotherapy after resection of TETs (22).

Contributions to the research topic on "Novel Treatments for Thymoma and Thymic Carcinoma" highlight recent developments in the understanding of the biology of TETs and review various aspects of management of TETs. Huang and colleagues describe previously unreported changes in the expression of apoptosis-related genes in WHO subtype B3 thymomas and thymic squamous cell carcinomas (23). These changes include up-regulation of the anti-apoptotic gene BIRC-3, 
overexpression of the BIRC-3 protein, and reduced expression of the pro-apoptotic gene, MTCH2 in thymic squamous cell carcinomas, and reduced expression of the pro-apoptotic gene, PMAIP1/NOXA in WHO subtype B3 thymomas. These discoveries have potential therapeutic implications since drugs targeting BIRC-3 and PMAIP-1 are in development (24).

Martinez and Browne review immunological deficiencies associated with thymoma and suggest a paradigm for comprehensive immunological evaluation in patients with thymoma, which should include an assessment of quantitative immunoglobulins, lymphocyte phenotyping, a vaccine challenge in patients suspected to have antibody deficiency and detection of anti-cytokine antibodies, whenever possible (25). Possible therapeutic interventions include immunoglobulin replacement in patients experiencing recurrent sinopulmonary infections due to immunoglobulin deficiency, and use of topical or systemic antifungal drugs in patients susceptible to chronic mucocutaneous candidiasis due to the presence of IL-17 or IL-22 antibodies (25).

Shapiro and Korst discuss the role of surgery for thymic tumors with pleural involvement (26). Surgical approaches that can be considered in this setting include metastasectomy for patients with a limited number of pleural lesions and extrapleural pneumonectomy for patients with more extensive pleural involvement. Data supporting the potential utility of intraoperative, hyperthermic, intrathoracic chemotherapy are also discussed and the need for prospective clinical trials to firmly establish the role of surgery for the management of stage IVA TETs is highlighted.

\section{REFERENCES}

1. Detterbeck FC. The creation of the international thymic malignancies interest group as a model for rare diseases. Am Soc Clin Oncol Educ Book (2012) 32:471-4. doi:10.14694/EdBook_AM.2012.32.471

2. Rajan A, Girard N, Marx A. State of the art of genetic alterations in thymic epithelial tumors. J Thorac Oncol (2014) 9:S131-6. doi:10.1097/JTO. 0000000000000298

3. Wang Y, Thomas A, Lau C, Rajan A, Zhu Y, Killian JK, et al. Mutations of epigenetic regulatory genes are common in thymic carcinomas. Sci Rep (2014) 4:7336. doi:10.1038/srep07336

4. Petrini I, Meltzer PS, Kim IK, Lucchi M, Park KS, Fontanini G, et al. A specific missense mutation in GTF2I occurs at high frequency in thymic epithelial tumors. Nat Genet (2014) 46:844-9. doi:10.1038/ng.3016

5. Gökmen-Polar Y, Cook RW, Goswami CP, Wilkinson J, Maetzold D, Stone JF, et al. A gene signature to determine metastatic behavior in thymomas. PLoS One (2013) 8:e66047. doi:10.1371/journal.pone.0066047

6. Evoli A, Minicuci GM, Vitaliani R, Battaglia A, Della Marca G, Lauriola L, et al. Paraneoplastic diseases associated with thymoma. J Neurol (2007) 254:756-62. doi:10.1007/s00415-006-0429-z

7. Holbro A, Jauch A, Lardinois D, Tzankov A, Dirnhofer S, Hess C. High prevalence of infections and autoimmunity in patients with thymoma. Hum Immunol (2012) 73:287-90. doi:10.1016/j.humimm.2011.12.022

8. Marx A, Hohenberger P, Hoffmann H, Pfannschmidt J, Schnabel P, Hofmann HS, et al. The autoimmune regulator AIRE in thymoma biology: autoimmunity and beyond. J Thorac Oncol (2010) 5:S266-72. doi:10.1097/JTO. 0b013e3181f1f63f

9. Meager A, Wadhwa M, Dilger P, Bird C, Thorpe R, Newsom-Davis J, et al. Anti-cytokine autoantibodies in autoimmunity: preponderance of neutralizing autoantibodies against interferon-alpha, interferon-omega and interleukin-12 in patients with thymoma and/or myasthenia gravis. Clin Exp Immunol (2003) 132:128-36. doi:10.1046/j.1365-2249.2003. 02113.x
The role of radiation therapy in the management of thymic epithelial tumors is reviewed by Komaki and Gomez (27). The indications for adjuvant and definitive radiation therapy are discussed as well as techniques to deliver radiation and the long-term effects of mediastinal radiation therapy.

Finally, the review by Chen and colleagues focuses on the latest advances in systemic therapies for TETs (28). Results from clinical trials evaluating novel biological therapies including histone deacetylase inhibitors, insulin-like growth factor inhibitors, and multikinase inhibitors are discussed, and ongoing phase II trials for TETs are highlighted.

The aforementioned manuscripts provide a snapshot of important research efforts related to TETs. Continued advances in the field have resulted in an ever increasing stream of data that offer newer insights into the biology of these rare tumors and support the use of newer paradigms of management for patients with thymoma and thymic carcinoma.

\section{AUTHOR CONTRIBUTIONS}

Conception and design: all authors; manuscript writing: all authors; final approval of manuscript: all authors.

\section{ACKNOWLEDGMENTS}

The authors acknowledge the Intramural Research Program of the $\mathrm{NIH}$, National Cancer Institute, Center for Cancer Research for their support.

10. Weksler B, Lu B. Alterations of the immune system in thymic malignancies. J Thorac Oncol (2014) 9:S137-42. doi:10.1097/JTO.0000000000000299

11. Burbelo PD, Browne SK, Sampaio EP, Giaccone G, Zaman R, Kristosturyan E, et al. Anti-cytokine autoantibodies are associated with opportunistic infection in patients with thymic neoplasia. Blood (2010) 116:4848-58. doi:10.1182/ blood-2010-05-286161

12. Browne SK. Anticytokine autoantibody-associated immunodeficiency. Annu Rev Immunol (2014) 32:635-57. doi:10.1146/annurev-immunol-032713120222

13. Vitiello L, Masci AM, Montella L, Perna F, Angelini DF, Borsellino G, et al. Thymoma-associated immunodeficiency: a syndrome characterized by severe alterations in NK, T and B-cells and progressive increase in naive CD8+ T cells. Int J Immunopathol Pharmacol (2010) 23:307-16.

14. Ahmad U, Huang J. Current readings: the most influential and recent studies involving surgical management of thymoma. Semin Thorac Cardiovasc Surg (2013) 25:144-9. doi:10.1053/j.semtcvs.2013.05.001

15. Girard N, Ruffini E, Marx A, Faivre-Finn C, Peters S; ESMO Guidelines Committee, et al. Thymic epithelial tumours: ESMO Clinical Practice Guidelines for diagnosis, treatment and follow-up. Ann Oncol (2015) 26(Suppl 5):v40-55. doi:10.1093/annonc/mdv277

16. Sakamaki Y, Oda T, Kanazawa G, Shimokawa T, Kido T, Shiono H. Intermediate-term oncologic outcomes after video-assisted thoracoscopic thymectomy for early-stage thymoma. J Thorac Cardiovasc Surg (2014) 148:1230-7.e1. doi:10.1016/j.jtcvs.2014.01.029

17. Schwartz GS, Yang SC. Robotic thymectomy for thymic neoplasms. Thorac Surg Clin (2014) 24:197-201,vii. doi:10.1016/j.thorsurg.2014.02.005

18. Mizuno T, Okumura M, Asamura H, Yoshida K, Niwa H, Kondo K, et al Surgical management of recurrent thymic epithelial tumors: a retrospective analysis based on the Japanese nationwide database. J Thorac Oncol (2015) 10:199-205. doi:10.1097/JTO.0000000000000378

19. Hamaji M, Ali SO, Burt BM. A meta-analysis of surgical versus nonsurgical management of recurrent thymoma. Ann Thorac Surg (2014) 98:748-55. doi: 10.1016/j.athoracsur.2014.04.028 
20. Rimner A. Postoperative radiotherapy: not all thymic malignancies are created equal. Cancer (2015) 121:972-4. doi:10.1002/cncr.29164

21. Ahmad U, Yao X, Detterbeck F, Huang J, Antonicelli A, Filosso PL, et al. Thymic carcinoma outcomes and prognosis: results of an international analysis. J Thorac Cardiovasc Surg (2015) 149:95-101. doi:10.1016/j.jtcvs.2014.09.124

22. Ruffini E, Guerrera F, Filosso PL; European Society of Thoracic Surgeons Thymic Working Group. Reply to Hamaji. Eur J Cardiothorac Surg (2015) 48:340-1. doi:10.1093/ejcts/ezu481

23. Huang B, Belharazem D, Li L, Kneitz S, Schnabel PA, Rieker RJ, et al. Antiapoptotic signature in thymic squamous cell carcinomas - functional relevance of anti-apoptotic BIRC3 expression in the thymic carcinoma cell line 1889c. Front Oncol (2013) 3:316. doi:10.3389/fonc.2013.00316

24. Ling X, Cao S, Cheng Q, Keefe JT, Rustum YM, Li F. A novel small molecule FL118 that selectively inhibits survivin, Mcl-1, XIAP and cIAP2 in a p53independent manner, shows superior antitumor activity. PLoS One (2012) 7:e45571. doi:10.1371/journal.pone.0045571

25. Martinez B, Browne SK. Good syndrome, bad problem. Front Oncol (2014) 4:307. doi: $10.3389 /$ fonc. 2014.00307
26. Shapiro M, Korst RJ. Surgical approaches for stage IVA thymic epithelial tumors. Front Oncol (2014) 3:332. doi:10.3389/fonc.2013.00332

27. Komaki R, Gomez DR. Radiotherapy for thymic carcinoma: adjuvant, inductive, and definitive. Front Oncol (2014) 3:330. doi:10.3389/fonc.2013.00330

28. Chen Y, Gharwan H, Thomas A. Novel biologic therapies for thymic epithelial tumors. Front Oncol (2014) 4:103. doi:10.3389/fonc.2014.00103

Conflict of Interest Statement: The authors declare that the research was conducted in the absence of any commercial or financial relationships that could be construed as a potential conflict of interest.

Copyright (c) 2015 Rajan, Wakelee and Giaccone. This is an open-access article distributed under the terms of the Creative Commons Attribution License (CC BY). The use, distribution or reproduction in other forums is permitted, provided the original author(s) or licensor are credited and that the original publication in this journal is cited, in accordance with accepted academic practice. No use, distribution or reproduction is permitted which does not comply with these terms. 